\title{
Mechanics of the Gyroscopic Precession and Calculation of the Galactic Mass
}

\author{
Jian Sun ${ }^{1 *}$, Ting Xu${ }^{1}$, Zheng Jiao', Hui Wang² \\ ${ }^{1}$ Shanghai University, Shanghai, China \\ ${ }^{2}$ Shanghai Advanced Research Institute, Chinese Academy of Sciences, Shanghai, China \\ Email: *sunjk12@gmail.com
}

How to cite this paper: Sun, J., Xu, T., Jiao, Z. and Wang, H. (2022) Mechanics of the Gyroscopic Precession and Calculation of the Galactic Mass. Journal of Modern Physics, 13, 34-45. https://doi.org/10.4236/jmp.2022.131003

Received: December 19, 2021

Accepted: January 17, 2022

Published: January 20, 2022

Copyright () 2022 by author(s) and Scientific Research Publishing Inc. This work is licensed under the Creative Commons Attribution International License (CC BY 4.0).

http://creativecommons.org/licenses/by/4.0/

\begin{abstract}
A spinning gyroscope precesses about the vertical due to a torque acting upon the wheel. The torque is generated by the shift of moment of force by gravity and it points to the vertical instead of the tangential direction of precession. This intuition offers an alternative and straightforward view of precession dynamics in comparison with the literature. It also presumes a dynamic balance of momentum between circular motions of the wheel spin and precession. Accordingly, the gyroscopic dynamics is then applied to the study of galactic motion of the solar system in space and the Galactic mass is calculated with the inclusion of gyroscopic effect of the solar planets. Results indicate that the gyroscopic effect of Mercury orbiting around the Sun can increase the calculated Galactic mass by $23 \%$ in comparison with the result obtained by the classic approach.
\end{abstract}

\section{Keywords}

Gyroscopic Precession, Solar System, Galactic Mass

\section{Introduction}

Newton's law of universal gravitation is arguably the first principle in astrophysics. It started with an apple falling down on his head and ended with the Moon falling sideways around Earth. Newton's comparison of acceleration of the apple to that of the Moon led to a rational understanding of the nature of gravity that came to be the fabric of our universe. All objects attract each other with a force that is proportional to the product of their masses and inversely proportional to their distance of separation. The laws of mechanics that govern the movement of objects on Earth also govern the motion of celestial bodies in space.

In the study of astrophysics, measuring the mass and motion of galaxies is one 
of our fundamental tasks. Luminous or baryonic matter in a galaxy such as stars and planets can be detected directly using optical and radio telescopes. And we can get an estimate of how much mass they contain. However, we cannot directly measure non-luminous matter, such as black holes that formed from stellar collapse, which we cannot see.

How can we measure the celestial mass that cannot be seen? According to the universal gravitational equation and Newton's second law of motion, if we know the mass of a body, we can work out how fast we need to go to orbit at distance. For instance, given the mass of the Sun and orbital radius of the solar planets, we can calculate the rotation curve of the solar system. Results show that the orbital or rotational speeds of the planets are inversely proportional to the square root of their orbital radii. The solar rotation curve falls. The calculation result is consistent with that of observation. Inversely, if we know the speed of a star orbiting on a circular orbit at distance from a galactic center, we can calculate the galactic mass interior to the orbit of the star. The gravitational forces from mass outside cancel out, assuming that the mass distribution is spherically symmetric. For in stance, given the orbital speed and radius of the Sun orbiting about the Galactic center, the Galactic mass can be calculated as an equivalent of 95 billion solar masses. Consequently, suppose we measure the speeds of stars at various radii from a galactic center. In theory, each star allows us to calculate the mass interior to the orbit of that particular star. If we observe many stars, we can map out the rotation curve of the galaxy.

Accordingly, when we apply the rotation curve to our Galaxy, we expect results similar to that of the solar system. Specifically, within the inner galaxy, enclosed mass rises, so does the rotation speed. There are no stars and no more mass beyond the optical edge of the Galaxy, and rotation speed supposedly falls. Instead, we observe a flat rotation curve for the Galaxy in which speed virtually stays the same as we move further away from the center [1] [2].

There can be two ways of reasoning with the observation, as we go back to the equation derived from Newton's universal gravitation and second law of motion. If orbital speed is constant with increasing orbital radius, then mass is proportional to radius. Hence, there ought to be more and more mass as we go to larger and larger distances from the center, implying that there can be a substantial amount of mass like dark matter we do not see in space [3] [4].

Alternatively, we can compare the patterns of motion between the solar planets and galactic stars like the Sun. A solar planet moves along with other planets in groups around the Sun, while the Sun moves with a group of its planets orbiting around it. Orbital rotation of the solar planets produces a combined angular motion that would keep the solar plane moving along a straight line due to the conservation of angular momentum. In other words, the solar system acts like a giant gyroscope in space. Does the law of conservation of angular momentum affect orbital motion of the solar system in our Galaxy? If so, how? Perhaps we want to go back to the basic physics of gyroscopes. 


\section{The gyroscope}

A gyroscope is made of a wheel or rotor on an axle that spins freely, usually mounted in a frame body. When spinning, the orientation of the spin axis is unaffected by tilting or rotating the body. Without its mounting body, a spinning gyroscope can stand by its axle like a top and move in counter-intuitive ways. It precesses about the vertical as if it defies gravity [5] [6] [7].

Newton's first law of motion tells us that a body in motion continues to move at a constant speed along a straight line unless acted upon by an unbalanced force. Since a spinning gyroscope precesses and changes its motion, there must be a net force acting upon it. And it has to be the force of gravity. Figure 1 shows a precessing gyroscope with a description of precession dynamics and geometric illustration of angular motion [8]. When the wheel is not spinning at the shown position, it simply topples over by turning to the right around the $\mathrm{x}$-axis, due to the moment of force created by its weight. When the wheel is spinning, it precesses by continuously falling sideways around the $z$-axis without actually falling over, tracing an invisible cone standing on its tip.

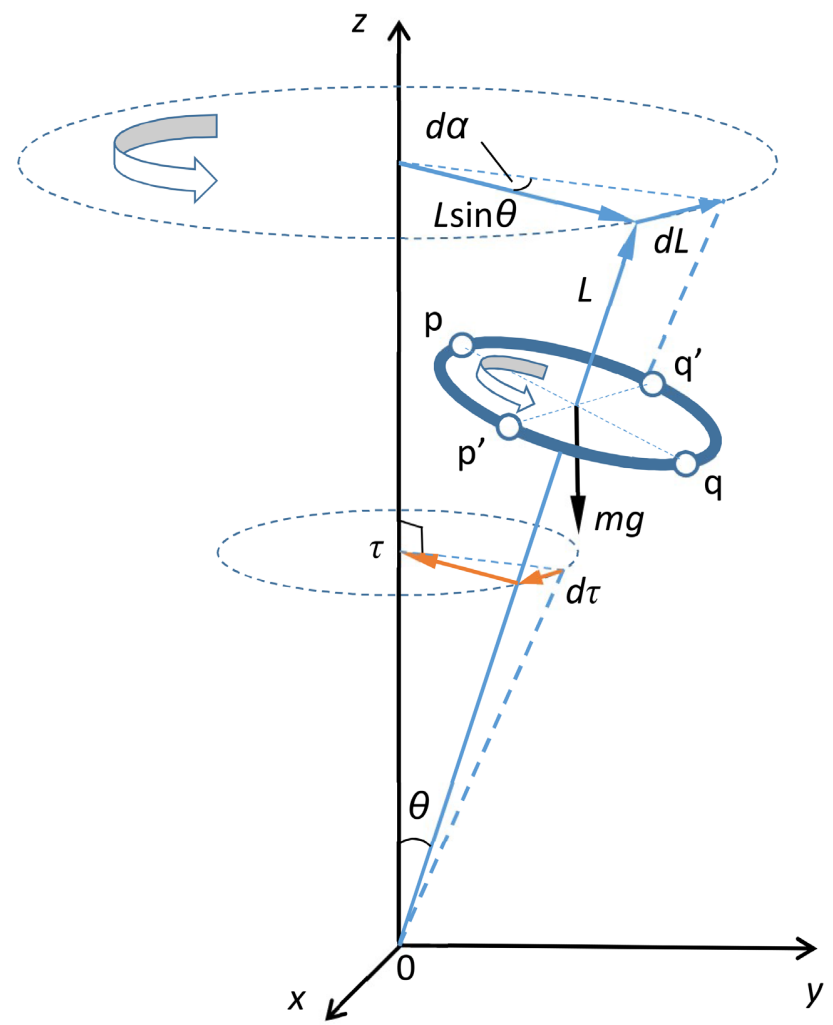

Figure 1. A spinning gyroscope falls sideways. The wheel consists of a solid ring connected to an axle with weightless spokes not shown. The gyroscope precesses counterclockwise as the wheel spins in the same direction. Positioned below the wheel for clarity, the torque of precession points to the z-axis due to a quarter-turn shift of the moment of force by gravity, according to the right-hand rule. The gyroscope will precess clockwise if the wheel changes its spin direction and the torque will be pointing away from the $\mathrm{z}$-axis. Note that angular momentum and torque change in opposing directions. It points to a balance of momentum in horizontal between the wheel spin and precession. 
How do we interpret precession? Assume our gyroscope is made of a solid ring for simplicity instead of a typical disk on an axle. Let's pick four points of mass $p, q, p^{\prime}$ and $q^{\prime}$ at top, bottom, left and right on the ring. Without spinning, all units of mass produce a total moment of force by gravity, with a moment arm being the distance from the wheel center of mass to the z-axis. This moment of force turns around the $p^{\prime}-q^{\prime}$ horizontal median and the wheel falls. When spinning counterclockwise, all mass units shift in position continuously and the wheel precesses counterclockwise around the $\mathrm{z}$-axis by turning around the $p$ - $q$ vertical median instead. It seems that the spinning of the wheel makes its turning shift by 90 degrees in the direction of precession. Since it is the force of gravity acting on the wheel that causes precession, the moment of force must also shift by 90 degrees due to spinning (see Appendix 1 for reasoning). In other words, the moment of force turns around the horizontal median without spinning. When spinning, it shifts by a quarter-turn counterclockwise and turns around the vertical median instead. The moment of force by gravity thus becomes the torque of precession, pointing to the $\mathrm{z}$-axis instead of the tangential direction of precession [8]. This quarter-turn shift of moment/torque drives the wheel to make a quarter-turn twist in falling: it falls sideways around its vertical median instead of around the horizontal. As it continues to fall sideways, the gyroscope precesses. And the faster the wheel spins, the quicker the mass units shift in position, and inversely the easier and slower it precesses.

Following the geometric description in Figure 1, the precession of the gyroscope and spin of the wheel can be described as,

$$
\begin{aligned}
& \mathrm{d} \alpha \cong \frac{\mathrm{d} L}{L \sin \theta} \\
& \mathrm{d} L=L \sin \theta \mathrm{d} \alpha
\end{aligned}
$$

where $\mathrm{d} \alpha$ and $\mathrm{d} L$ are the changes of precession angle and angular momentum. $\theta$ is the tilt angle of the gyroscope from the vertical. The torque of precession that changes the angular momentum can be formulated as,

$$
\tau=\frac{\mathrm{d} L}{\mathrm{~d} t}=\frac{L \sin \theta \mathrm{d} \alpha}{\mathrm{d} t}=L \sin \theta \omega_{p}
$$

where $\tau$ is the torque and $\omega_{p}$ is the rate of precession. Calculation of the precession rate is derived in Appendix 1.

In general, without a net force acting on a spinning gyroscope, the orientation of its spin axis remains unchanged. When standing by its axle, gravity force exerts on the wheel and turns into a shifting moment for precession. And the wheel changes its orientation by turning around its vertical median. When it completes a full circle of precession, it also finishes a complete cycle of orientation change at a constant speed. It is a result of the dynamic balance of momentum between the wheel spin and precession.

\section{The Solar System}

Suppose our solar system revolves around the Galactic center (GC) in a way sim- 
ilar to gyroscopic precession. Planets including Earth rotate around the Sun to form the spinning solar plane, which orbits about the GC or precesses like a gyroscope wheel around the vertical, as shown in Figure 2 in three descriptive orbital positions. In fact, instead of a standing wheel, a spinning bicycle wheel in suspension is a better analogy for the solar scenario. The gyroscopic mechanism is the same regardless. Using Earth as an example, the rotation of Earth around the Sun generates an angular momentum pointing to the upper z-axis. The change of precession angle of the solar plane and angular momentum of the solar orbiting Earth follows the gyroscopic model,

$$
\begin{aligned}
& \mathrm{d} \alpha \cong \frac{\mathrm{d} L}{L \sin \beta} \\
& \mathrm{d} L=L \sin \beta \mathrm{d} \alpha
\end{aligned}
$$

where $\mathrm{d} \alpha, L$, and $\beta$ are the change of precession angle, angular momentum of the solar orbiting Earth, and tilt angle of the solar plane from the Galactic plane. The torque that changes the angular momentum is formulated as,

$$
\tau=\frac{\mathrm{d} L}{\mathrm{~d} t}=\frac{L \sin \beta \mathrm{d} \alpha}{\mathrm{d} t}=L \sin \beta \omega_{p}
$$

where $\omega_{p}$ is the precession rate of Earth or solar plane about the GC, and $\tau$ is the torque of precession. Earth is treated as a point of mass in our study so the angular momentum generated by its spin around its polar axis is ignored.

When the solar plane completes a Galactic circle, it also finishes a circle of precession around the $\mathrm{z}$-axis and a complete horizontal turn by itself around a

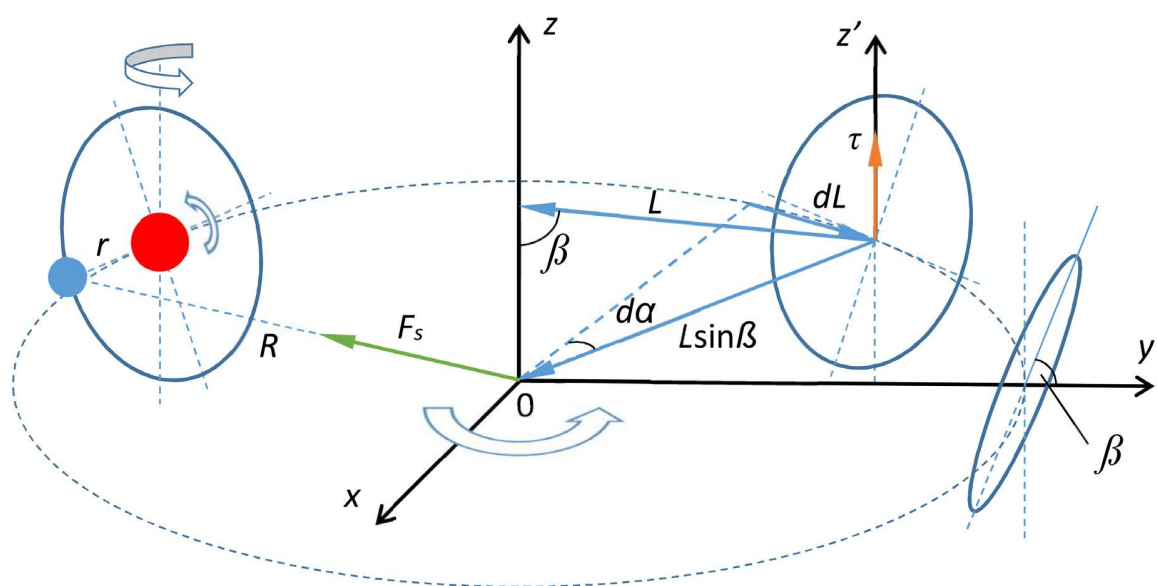

Figure 2. The spinning solar plane orbits or precesses about the GC in three select positions: Earth rotating around the Sun in the solar plane in the Galactic plane on the left; angular momentum of the solar orbiting Earth changing with its precession angle on the middle right; solar plane tilting from the Galactic plane on the right. The front side of the solar plane faces the $\mathrm{z}$-axis at all times. Both the solar plane spin and Galactic rotation are shown counterclockwise for a clear geometric view of vectors. The torque of precession points upward, according to the right-hand rule. It will point downward if the solar system revolves clockwise around the GC. In space, the gravitational pull from Galaxy points to the GC, rather than downward, which is different from the case of the gyroscope on Earth in Figure 1. 
moving $z^{\prime}$-axis that goes through the solar center (Figure 2), just like a precessing gyroscope. This forced horizontal turn or inclination change of the solar plane is presumably due to an additional gravitational pull from the GC. Without this gravitational pull, the solar plane would turn its side to face the center in every half of its Galactic circle due to its conservation of angular momentum. This assumes that the solar plane keeps orbiting in the Galaxy as a point of mass, which is the implication in conventional calculations of the Galactic mass. It may not be a reasonable assumption as the solar planets would experience a substantial gravitational change when the solar plane moves into positions where it is aligned with the GC: the Galactic gravitational force becomes too strong when the planets position between the Sun and GC; the force is too weak when they reverse their positions with the Sun. Substantial gravitational changes as such can affect and even break the integrity of the solar system.

Likewise, assuming the solar orbit of Earth effectively forms a ring of uniform mass distribution in its rotation around the Sun, this Earth orbit-ring (EOR) completes a horizontal turn by itself around the moving $\mathrm{z}^{\prime}$-axis in one Galactic cycle. Therefore, a torque must be acting on the EOR during precession, which is generated by the (additional) Galactic gravitational pull. Let's call it the action torque. It exerts on the EOR so that it cannot turn its side while facing the GC in precession. In response, the EOR has to generate a reaction torque by its angular momentum to counterbalance the action torque. This results in a dynamic balance of torque on the EOR between its precession in circular motion and inertia of moving straight. Unlike gyroscopes on Earth where gravity is the lone force to drive precession, the inertia of the EOR drives precession along with the gravitational pull in space. And the precession direction of the EOR is untethered from its spin direction. When two torques are equally matched, so are their corresponding forces in opposing directions. The action force that produces the torque of precession must be balanced in horizontal with the reaction force generated by the angular momentum. And the latter can be formulated as a force pulling away from the GC, as shown in Figure 2,

$$
F_{s}=\frac{\tau}{r}=\frac{L \omega_{p} \sin \beta}{r}
$$

where $F_{s}$ is the force of solar orbiting Earth and $r$ is the solar orbital radius of Earth. This reaction force generated by the angular momentum of the EOR must be overcome in its Galactic motion and subtracted in calculating the Galactic mass using Newton's law of circular motion as follows,

$$
F-F_{s}=m a=m \frac{v^{2}}{R}
$$

where $F$ is the gravitational force of the GC, $m, a, v$ and $R$ are the mass, Galactic centripetal acceleration, Galactic orbital speed and Galactic orbital radius (distance to the GC) of Earth. Combining with the equation of universal gravitation,

$$
\frac{G M m}{R^{2}}-\frac{L \omega_{p} \sin \beta}{r}=\frac{m v^{2}}{R}
$$


where $G$ is the universal gravitational constant and $M$ is the Galactic mass or mass of bodies interior to Galactic orbit of the solar system. Thus $M$ can be calculated as,

$$
M=\frac{R v^{2}}{G}+\frac{R^{2} L \omega_{p} \sin \beta}{r G m}
$$

It reduces down to the conventional equation (the first term on the right) when the angular momentum of the EOR is treated as zero (in the second term on the right). The angular momentum can be estimated by assuming the solar orbiting Earth as a ring of uniform mass distribution.

$$
L=I \omega=m r^{2} \omega
$$

where $I$ and $\omega$ are the moment of inertia and spin speed of the EOR. The mass of Earth is canceled out in calculation after combining Equations (8) and (9).

\section{Discussion}

In general, the Galactic mass can also be calculated with other solar planets in a similar fashion or with the Sun as a solid sphere, assuming that the Sun's spin axis points to the z-axis. It is rather ideal to treat an orbiting body as a ring of uniform mass in the calculation. In reality, orbital motion of the solar planets produces a non-uniform distribution of their angular momenta, presumably causing oscillations within the solar plane. The derivation above is to find the Galactic mass with the inclusion of gyroscopic effect. Inversely, given a galactic or solar mass, Equation (7) can also be used to calculate the potential orbital speed increase of stars or solar planets due to the gyroscopic effect of the planets or moons (Appendix 2).

Results of the Galactic mass increase calculated with gyroscopic motion of the solar planets and the Sun are presented in Table 1, shown as a list of mass calculated by the second term on the right in Equation (8). Table 2 lists the parameters and constants used in the calculation. It appears that the gyroscopic effect falls with the solar orbital radius, presumably due to the decreasing solar gravitational pull that causes a decrease in the solar orbital speed. To calculate the total solar gyroscopic effect, the solar system can be approximated as a monolithic rigid plane of mass by adding up the angular momenta of all the solar bodies. However, estimation of the average radius and angular speed of the plane becomes a complicated issue. Hence, the gyroscopic effect is calculated and presented separately with each solar body in this study.

The Sun has a minimal gyroscopic effect on assessing the Galactic gravitational and mass increase as shown in Table 1, which is reasonable due to its small angular momentum. Among solar planets, Mercury has the highest precession impact on the Galactic gravitational increase that results in an additional 22 billion solar masses from the value of 95 billion solar masses obtained by the classic approach. It suggests that the effective force of gyroscopic precession of the solar 
Table 1. Galactic mass increase calculated with the gyroscopic motion of each solar body in the solar system.

\begin{tabular}{cccc}
\hline & $\begin{array}{c}\text { Orbital radius } \\
\text { m }\end{array}$ & $\begin{array}{c}\text { Orbital period } \\
\text { days }\end{array}$ & $\begin{array}{c}\text { Solar mass equivalent, } \\
\text { billion }\end{array}$ \\
\hline Mercury & $5.79 \times 10^{10}$ & 88 & 22.1 \\
Venus & $1.08 \times 10^{11}$ & 224.7 & 16.1 \\
Earth & $1.50 \times 10^{11}$ & 365.2 & 13.7 \\
Mars & $2.28 \times 10^{11}$ & 687 & 11.1 \\
Jupiter & $7.79 \times 10^{11}$ & 4331 & 6.03 \\
Saturn & $1.43 \times 10^{12}$ & 10,747 & 4.47 \\
Uranus & $2.87 \times 10^{12}$ & 30,589 & 3.15 \\
Neptune & $4.50 \times 10^{12}$ & 59,800 & 2.52 \\
Pluto & $5.91 \times 10^{12}$ & 90,560 & 2.19 \\
Sun & $6.96 \times 10^{5}$ & 27 & 0.00042 \\
\hline
\end{tabular}

Table 2. Calculation parameters and constants.

$\begin{array}{cc}R, \mathrm{~m} & 2.60 \times 10^{20} \\ V, \mathrm{~m} / \mathrm{s} & 2.20 \times 10^{5} \\ \omega_{p}, \mathrm{rad} / \mathrm{s} & 9.06 \times 10^{-16} \\ G, \mathrm{Nm}^{2} / \mathrm{kg}^{2} & 6.67 \times 10^{-11} \\ \text { Solar mass, } \mathrm{kg} & 1.99 \times 10^{30} \\ \beta, \mathrm{rad} & \pi / 2\end{array}$

plane is comparable in magnitude to the gravitational force calculated by the classic approach. In other words, it is reasonable to assume that our solar system revolves around the GC like a precessing gyroscope as shown in Figure 2, instead of being a point of mass as implied in the classic approach.

\section{Concluding Remarks}

A new mechanical model of gyroscopic precession is derived and applied to the study of galactic motion of the solar system in space. The Galactic mass is calculated with the inclusion of gyroscopic effect of the solar planets. Results indicate that the gyroscopic effect of Mercury orbiting around the Sun can increase the calculated Galactic mass by $23 \%$ in comparison with the result obtained by the classic approach. In comparison with other theories, the maximal percentage increase of the Galactic mass calculated by the gyroscopic effect in this study is an order of magnitude smaller than those obtained by the dark matter models [4]. Nevertheless, the effect of gyroscopic precession introduces an empirical variable in the study of astrophysics. It can potentially lead to new understandings of our Galaxy. 


\section{Acknowledgments}

Planetary data are obtained from NASA and Wikipedia websites. Financial support is graciously provided by Shanghai University. All calculations are performed with Microsoft Excel.

\section{Conflicts of Interest}

The authors declare no conflicts of interest regarding the publication of this paper.

\section{References}

[1] Palma, C. (2020) ASTRO 801: Planets, Stars, Galaxies, and the Universe, Open Educational Resources Initiative of John A. Dutton e-Education Institute, The Pennsylvania State University. https://www.e-education.psu.edu/astro801/content/18_p8.html

[2] Kuhfittig, P. and Gladney, V. (2014) Journal of Modern Physics, 5, 1931-1937. https://doi.org/10.4236/jmp.2014.517187

[3] Mongan, T. (2017) Journal of Modern Physics, 8, 919-922. https://doi.org/10.4236/jmp.2014.517187

[4] Karukes, E.V., Benito, M., Iocco, F., Trotta, R. and Geringer-Sameth, A. (2020) Journal of Cosmology and Astroparticle Physics, 5, 33. https://doi.org/10.1088/1475-7516/2020/05/033 https://phys.org/news/2019-12-mass-milky-billion-sun.html

[5] Deimel, R.F. (1950) Mechanics of the Gyroscope. Dover Publications, New York.

[6] Scarborough, J.B. (2014) The Gyroscope Theory and Applications. Nabu Press, London.

[7] Usubamatov, R. (2021) World Journal of Mechanics, 11, 177-185. https://doi.org/10.4236/wjm.2021.119013

[8] Ling, S.J., Sanny, J. and Moebs, B. (2020) 11.5 Precession of a Gyroscope-University Physics Volume 1, Licensed by OpenStax University Physics.

https://openstax.org/books/university-physics-volume-1/pages/11-4-precession-of-agyroscope 


\section{Notations}

$\alpha, \mathrm{d} \alpha$-precession angle and its change

$L, \mathrm{~d} L$-spin angular momentum and its change

$\theta$-tilt angle of gyroscope from the vertical

$\tau$-torque of precession

$\omega_{p}$-rate of precession

$\beta$-tilt angle of the solar plane from the Galactic plane

$F_{s}$-force by the angular momentum change of EOR

$F$-gravitational force of the GC

$m$-mass of the gyroscope wheel or Earth

a-Galactic centripetal acceleration of Earth

$V$-Galactic orbital speed of Earth

$R$ - Galactic orbital radius of Earth

$G$-universal gravitational constant

$M$-Galactic mass

$r$-radius of the gyroscope wheel or EOR

I-moment of inertia

$\omega$-rate of spin

mg-weight of the gyroscope wheel

$h$-length of the gyroscope axle 


\section{Appendix 1. Interpretation of the Shift of Moment of Force and Calculation of the Precession Rate}

As the wheel tends to turn around the $p^{\prime}-q^{\prime}$ horizontal median in precession due to the moment of force by gravity, the mass of the ring generates transversal acceleration. The upper half of the ring accelerates upward and the lower half does downward with the max acceleration at $p$ and $q$. All mass units accelerate and travel along the ring counterclockwise. Unit $p$ accelerates upward while traveling to $p^{\prime}$ and decelerates while continuing to $q$. Consequently, its upward transversal velocity reaches a max at $p^{\prime}$ and reduces back to zero at $q$. Similarly, unit $q$ accelerates downward while traveling to $q^{\prime}$ and decelerates while continuing to $p$. Its downward transversal velocity reaches a max at $q$ ' and reduces back to zero at $p$. All mass units experience such a cycle of transversal velocity delay/shift for a quarter-turn from acceleration. As a result, the potential of the wheel turning around the $p^{\prime}-q^{\prime}$ horizontal median becomes turning around the $p-q$ vertical median instead. And the shift of transversal velocity practically twists the moment of force by a quarter-turn counterclockwise.

A precessing gyroscope does three circular moves at the same time: spinning the wheel, turning around its vertical median, and revolving around the vertical axis. The first movement makes the second that drives the third. Mechanically, the torque of precession comes from the force of gravity by twisting the moment/torque via shifting mass units on the spinning wheel. Following Equation (2),

$$
L \omega_{p} \sin \theta=\tau=m g h \sin \theta
$$

where $m g$ and $h$ are the weight of the wheel and length of the axle. Hence, the precession rate becomes,

$$
\omega_{p}=\frac{m g h}{L}
$$

where $L$ is the angular momentum of the spin wheel, which can be calculated based on the wheel shape, a solid ring in this study,

$$
L=I \omega=m r^{2} \omega
$$

where $I$ and $\omega$ are the moment of inertia and spin rate of the wheel.

\section{Appendix 2. Calculation of Orbital Speed Due to Gyroscopic Effect}

Equation (7) is reformulated by replacing the reaction force created by the angular momentum with the action force that creates the torque of precession,

$$
\frac{G M m}{R^{2}}+\frac{L \omega_{p} \sin \beta}{r}=\frac{m v^{2}}{R}
$$

Plug in the moment of inertia, replace the angular speed of precession with the linear speed of precession, and let $\beta=\pi / 2$,

$$
R v^{2}-r \omega R v-G M=0
$$


Solve for $v$. The potential orbital speed increase of Earth due to the gyroscopic effect of the Moon is calculated accordingly. Without changing its orbital radius and flying away from Earth, the Moon would need to increase its orbital speed by more than 130 thousand times in order to increase the orbital speed of Earth by $1 \%$, provided that the Moon also changes the inclination of its orbit to squarely face the Sun. In other words, the gyroscopic effect of the Moon is negligible in comparison with those of the solar planets. 